\title{
Empiema paraneumónico: caracterización y factores asociados a morbi-mortalidad en 242 casos tratados quirúrgicamente
}

\author{
ROBERTO GONZÁLEZ L.*,**, RAFAEL PRATS M.*,**, DAVID LAZO P.*,**, \\ ANDRÉS JADUE T.**, GERARDO MORDOJOVICH R.*,**, \\ RAIMUNDO SANTOLAYA C. ${ }^{* * * *}$ y PATRICIO RODRÍGUEZ D.*,**
}

Parapneumonic empyema: characterization and factors associated with morbidity and mortality in 242 surgical cases

Objectives: To describe and evaluate factors associated with morbidity and mortality in surgically treated parapneumonic empyemas (PNE). Method: Retrospective review between January 2000 and August 2006. We described clinical features and we performed univariate and multivariate analysis to find the factors associated with morbidity and mortality. SPSS 15.0 program was used in the statistical analysis. Results: 242 of 343 surgically treated empyemas (70.6\%) were paraneumonic. Of these 165 (68.2\%) were men, average age 52.1 years, 229 (94.6\%) were community-acquired pneumonia (CAP) and 13 (5.4\%) nosocomial. Germs were isolated in pleural fluid in 57 (23.6\%). Surgical procedures were: 183 (75.6\%) decortications, 49 (20.2\%) pleurotomies, 7 (2.9\%) video-assisted surgery and 3 (1.2\%) decortications with pulmonary resection. Complications occurred in 65 cases (26.9\%) and 16 patients died (6.6\%). We found variables associated with morbidity and mortality. Conclusions: PNE is the most common cause of empyema, mostly associated with CAP and germs are difficult to identify. Decortication is the most common surgical treatment. Morbidity and mortality are present. Variables associated with morbidity and mortality were identified in this clinical series.

Key words: Empyema, pleural diseases, pneumonia, thoracic surgical procedures, morbidity, mortality.

\section{Resumen}

Objetivos: Describir las características y evaluar los factores asociados a morbi-mortalidad en el empiema paraneumónico (EPN) tratado quirúrgicamente. Método: Revisión retrospectiva entre Enero 2000 y Agosto 2006. Se describen características y factores asociados a morbi-mortalidad, se realizó análisis univariado y multivariado utilizando programa SPSS 15.0. Resultados: 242 pacientes tenían EPN (70,6\% de los empiemas tratados), 165 (68,2\%) fueron hombres, edad promedio 52,1 años, 229 (94,6\%) fueron neumonías adquiridas en la comunidad (NAC) y 13 (5,4\%) intrahospitalarias. Se aisló gérmenes en líquido pleural en 57 (23,6\%). Se realizaron 183 (75,6\%) decorticaciones, 49 (20,2\%) pleurotomías, 7 (2,9\%), cirugías video-asistidas y 3 (1,2\%) decorticaciones con resección pulmonar. Presentaron complicaciones 65 (26,9\%). Fallecieron 16 pacientes (6,6\%). Se identificaron variables asociadas a morbi-mortalidad. Conclusiones: El EPN es la causa más frecuente de empiema, la mayoría está asociado a NAC y en pocos se identifican gérmenes. La decorticación es el procedimiento más frecuente. Se identificaron las variables asociadas a morbi-mortalidad en esta serie clínica.

Palabras clave: Empiema pleural, patología pleural, neumonía, cirugía torácica, morbilidad, mortalidad.

No existen conflictos de interés ni apoyo financiero extrainstitucional.

* Sección de Cirugía de Tórax, Servicio Médico Quirúrgico Respiratorio, Instituto Nacional del Tórax.

** Departamento de Cirugía, Campus Oriente, Universidad de Chile. 


\section{Introducción}

El empiema paraneumónico (EPN) corresponde aproximadamente al $60 \%$ a $70 \%$ de los casos totales de empiema pleural ${ }^{1-3}$. Un hecho conocido es que, entre el 20 a $40 \%$ de las neumonías evolucionan con derrame pleural, pero sólo un bajo porcentaje de éstas, evolucionan con empiema pleural $^{4-6}$.

El EPN se considera una grave complicación de la neumonía. En Estados Unidos se diagnostican aproximadamente 60.000 casos por año y la mortalidad alcanza el $20 \%$ en grupos de riesgo $^{2,4,5}$. Es más frecuente en edades extremas y existen factores de riesgo para su desarrollo como inmunosupresión, pacientes con infección por VIH, diabetes mellitus, enfermedad pulmonar obstructiva crónica, alcoholismo y aspiración bronquial entre otros ${ }^{5,7,8}$.

Durante el siglo pasado se produjo una significativa disminución en el número de casos, así como en la morbilidad y mortalidad asociada a EPN. Sin embargo, en la última década éstas se han mantenido estacionarias, incluso han aumentado en algunos subgrupos de riesgo, todo esto a pesar de los progresos constantes en terapia antibiótica, técnicas quirúrgicas y cuidados post operatorios ${ }^{9,10}$.

Los objetivos de nuestra comunicación son caracterizar y evaluar factores asociados a morbimortalidad de EPN tratados quirúrgicamente.

\section{Material y Métodos}

Se realizó una revisión retrospectiva de bases de datos de empiema, de protocolos operatorios y del registro de pabellón del Instituto Nacional del Tórax. El período analizado comprendió desde enero de 2000 hasta agosto de 2006.

Se incluyeron todos los pacientes con diagnóstico de EPN y en quienes se realizó algún procedimiento quirúrgico.

Se consideraron EPN los casos que cumplían con los 2 criterios siguientes:

A. Presencia de una o más de las siguientes características en el contenido de la ocupación pleural:

- Cultivo microbiológico o tinción de Gram positivos.

- $\mathrm{pH}<7,2$.

- Hallazgos macroscópicos compatibles con empiema.

B. Proceso secundario a neumonía.
Se registró: género, edad, procedencia (lugar donde se diagnosticó y trató la neumonía antes de ser derivado para el procedimiento quirúrgico), comorbilidad, tipo de neumonía (al momento del diagnóstico de ésta), cultivos microbiológicos, microorganismos aislados, procedimientos quirúrgicos (se consideró el procedimiento final o definitivo) y morbi-mortalidad. Para evaluar factores asociados a morbi-mortalidad se seleccionaron 26 variables, se realizó análisis univariado y multivariado cuya variable dependiente fue morbilidad y mortalidad por separado. Se utilizó el programa SPSS 15.0. Se consideró significativo un valor de $\mathrm{p}<0,05$.

\section{Resultados}

Durante el período estudiado, en el Instituto Nacional del Tórax se realizaron procedimientos quirúrgicos en 343 pacientes con empiema pleural. De éstos, 242 fueron en pacientes con EPN, lo que equivale al $70,6 \%$ del total de los empiemas pleurales.

La mayoría de los 242 pacientes con EPN fueron de género masculino (relación de 2,1:1) y el promedio de edad fue de $52,16 \pm 17,47$, con un rango entre los 15 a 86 años (Tabla 1).

En relación a la procedencia de los pacientes: sólo 12 (4,8\%) fueron del Instituto Nacional del Tórax y 230 (95,2\%) fueron derivados desde otros hospitales. De los derivados, 192 (79,3\%) provenían de otros hospitales de la Región Metropolitana y 38 (15,9\%) de hospitales de otras regiones de Chile.

Las comorbilidades de los pacientes se describen en la Tabla 2. Destacamos que $18(7,4 \%)$ pacientes tenían EPN en un "ambiente neoplásico", es decir, en pacientes con alguna neoplasia maligna, ya sea pleuropulmonar o de otro tipo. Además, se encontraron factores asociados a gra-

Tabla 1. Distribución según género y edad de 242 pacientes con Empiema paraneumónico tratados quirúrgicamente

\begin{tabular}{|lcr|}
\hline Distribución & n & $(\%)$ \\
Género & \multicolumn{2}{c|}{} \\
Masculino & 165 & $(68,2 \%)$ \\
Femenino & 77 & $(31,8 \%)$ \\
Relación M / F & $2,1 / 1$ & \\
Edad (años) & \multicolumn{2}{c}{$52,16 \pm 17.47$} \\
Promedio & \multicolumn{2}{c}{53} \\
Mediana & \multicolumn{2}{c}{$15-86$} \\
Rango & \multicolumn{2}{c}{5} \\
\hline
\end{tabular}


Tabla 2. Comorbilidades y factores asociados a gravedad en 242 pacientes con empiema paraneumónico tratados quirúrgicamente

\begin{tabular}{|lrr|}
\hline & n & \% \\
Comorbilidad & & \\
Tabaquismo & 90 & 37,2 \\
Hipertensión arterial & 67 & 27,7 \\
Diabetes mellitus & 48 & 19,8 \\
Ambiente neoplásico & 18 & 7,4 \\
\hline Factores asociados a gravedad & & \\
Deterioro de conciencia & 27 & 11,2 \\
Deterioro hemodinámico & 21 & 8,7 \\
\hline
\end{tabular}

Tabla 4. Estudios microbiológicos en 242 pacientes con empiema paraneumónico tratados quirúrgicamente

\begin{tabular}{|lrr|}
\hline Estudio microbiológico & n & \% \\
\hline Microorganismos aislados* & & \\
$\quad$ Positivo & 137 & 56,6 \\
$\quad$ Negativo & 105 & 43,4 \\
Cultivo de líquido pleural & & \\
$\quad$ Negativo & 185 & 76,4 \\
$\quad$ Positivo & 57 & 23,6 \\
\hline Cultivo polimicrobiano & 16 & 6,6 \\
\hline
\end{tabular}

*Germen aislado en cualquier tipo de cultivo: hemocultivo, expectoración o líquido pleural.

Tabla 5. Microorganismos aislados en estudios microbiológicos de líquido pleural en 242 pacientes con empiema paraneumónico tratados quirúrgicamente

\begin{tabular}{|lrc|}
\hline Microorganismos & n & \% \\
\hline Staphylococcus aureus & 42 & 17,4 \\
\hline Streptococcus spp & 27 & 11,2 \\
Pseudomonas aeruginosa & 13 & 5,4 \\
Klebsiella pneumoniae & 9 & 3,7 \\
Escherichia coli & 9 & 3,7 \\
Acinectobacter baumannii & 6 & 2,5 \\
Otros & 8 & 3,3 \\
\hline
\end{tabular}

Tabla 3. Tipo de neumonía (al momento del diagnóstico de ésta) en 242 pacientes con empiema paraneumónico tratados quirúrgicamente

\begin{tabular}{|lrc|}
\hline Tipo Neumonía & n & \% \\
\hline Intrahospitalaria & 13 & 5,4 \\
Adquirida en la comunidad & 229 & 94,6 \\
ATS*: I & 0 & - \\
$\quad$ II & 23 & 9,6 \\
$\quad$ III & 201 & 83 \\
$\quad$ IV & 5 & 2 \\
Otras características descritas & & \\
Necrotizante & 8 & 3,3 \\
Bilateral & 3 & 1,2 \\
Por aspiración & 3 & 1,2 \\
\hline
\end{tabular}

*ATS: Clasificación de la American Thoracic Society.

vedad al momento del procedimiento quirúrgico, como deterioro de conciencia y deterioro hemodinámico (requerimiento de drogas vaso activas).

El tipo de neumonía fue: en 229 (94,6\%) adquirida en la comunidad (NAC) y en $13(5,4 \%)$ intrahospitalaria (NIH) (Tabla 3).

Se aisló algún microorganismo en cualquier tipo de cultivo (hemocultivo, expectoración o líquido pleural) en 137 (56,6\%) pacientes y en líquido pleural en 57 (23,6\%), de los cuales 16 $(6,6 \%)$ fueron polimicriobianos (Tabla 4). El germen más frecuentemente aislado fue el Staphylococcus aureus (Tabla 5).

Se realizaron $183(75,6 \%)$ decorticaciones por toracotomía, 49 (20,2\%) pleurotomías, 7 (2,9\%) cirugías videotoracoscópicas o video-asistidas y $3(1,2 \%)$ decorticaciones con resección pulmonar (Tabla 6).

Presentaron complicaciones 65 (26,9\%) pacientes, se reoperaron $16(6,6 \%)$ y $22(9,1 \%)$ requirieron unidad de cuidados intensivos (UCI) en el post operatorio (Tablas 6 y 7).

Fallecieron $16(6,6 \%)$ pacientes, todos por sepsis.

De las variables seleccionadas para analizar la morbilidad presentaron significación estadís-

Tabla 6. Procedimientos quirúrgicos y morbi-mortalidad según tipo de procedimiento en 242 pacientes con empiema paraneumónico tratados quirúrgicamente

\begin{tabular}{|lccc|}
\hline Procedimiento quirúrgico & Total (\%) & Morbilidad (\%) & Mortalidad (\%) \\
Decorticación por toracotomía & $183(75,6)$ & $55(30)$ & $8(4,4)$ \\
Pleurotomía & $49(20,2)$ & $9(18,4)$ & $8(16,3)$ \\
Toracoscópico (videotoracoscopía o video-asistida) & $7(2,9)$ & 0 & 0 \\
Decorticación más resección pulmonar & $3(1,2)$ & $1(33,3)$ & 0 \\
Total & $242(100)$ & $65(26,9)$ & $16(6,6)$ \\
\hline
\end{tabular}


Tabla 7. Complicaciones, reoperaciones y requerimientos de UCI post operatoria en 242 pacientes con empiema paraneumónico tratados quirúrgicamente

\begin{tabular}{|lrr|}
\hline Complicaciones más frecuentes & n & \% \\
Empiema (colección residual) & 17 & 7,0 \\
Fuga aérea & 7 & 2,9 \\
Hemotórax & 4 & 1,7 \\
Insuficiencia respiratoria & 4 & 1,7 \\
Infección herida operatoria & 3 & 1,2 \\
Sepsis de origen extrapleural & 11 & 4,5 \\
Reoperaciones & 16 & 6,6 \\
$\quad$ Empiema (colección residual) & 12 & 4,9 \\
$\quad$ Sangrado & 4 & 1,7 \\
Requerimiento de UCI post operatoria & & \\
$\quad$ & 220 & 90,9 \\
$\quad$ No & 22 & 9,1 \\
\hline
\end{tabular}

tica en el análisis multivariado: identificación de germen, cultivo con Staphylococcus aureus, cultivo con Klebsiella pneumoniae, necesidad de reoperación y necesidad de UCI. De las variables seleccionadas para la mortalidad presentaron significación estadística en el análisis multivariado: edad mayor de 70 años, comorbilidad neoplásica, diabetes mellitus, deterioro de conciencia, deterioro hemodinámico, identificación de germen, cultivo con Klebsiella pneumoniae, cultivo con Acinetobacter baumannii y necesidad de UCI (Tabla 8).

\section{Comentario}

Esta serie está compuesta por pacientes con EPN en quienes se realizó un procedimiento quirúrgico por cirujano, que va desde la pleu-

Tabla 8. Análisis univariado y multivariado de variables seleccionadas como factores asociados a morbi-mortalidad en 242 pacientes con empiema paraneumónico tratados quirúrgicamente

\begin{tabular}{|c|c|c|c|c|}
\hline Variables & $\begin{array}{l}\text { Morbilidad } \\
\text { univariado }\end{array}$ & $\begin{array}{c}\text { Morbilidad } \\
\text { multivariado }\end{array}$ & $\begin{array}{l}\text { Mortalidad } \\
\text { univariado }\end{array}$ & $\begin{array}{l}\text { Mortalidad } \\
\text { multivariado }\end{array}$ \\
\hline Edad $<$ o $>70$ años & NS & NS & $\mathrm{p}<0,05$ & $\mathrm{p}<0,05$ \\
\hline Género M o F & NS & NS & NS & NS \\
\hline Ambiente neoplásico & NS & NS & $\mathrm{p}<0,05$ & $\mathrm{p}<0,05$ \\
\hline Diabetes mellitus & NS & NS & $\mathrm{p}<0,05$ & $\mathrm{p}<0,05$ \\
\hline Tabaquismo & NS & NS & NS & NS \\
\hline Fiebre & NS & NS & NS & NS \\
\hline Deterioro hemodinámico & $\mathrm{p}<0,05$ & NS & $\mathrm{p}<0,05$ & $\mathrm{p}<0,05$ \\
\hline Deterioro de conciencia & $\mathrm{p}<0,05$ & NS & $\mathrm{p}<0,05$ & $\mathrm{p}<0,05$ \\
\hline Taquicardia & NS & NS & NS & NS \\
\hline NAC o NIH & NS & NS & NS & NS \\
\hline ATS II, III, IV & NS & NS & NS & NS \\
\hline Neumonía bilateral & NS & NS & NS & NS \\
\hline Neumonía necrotizante & NS & NS & NS & NS \\
\hline Germen aislado & $\mathrm{p}<0,05$ & $\mathrm{p}<0,05$ & $\mathrm{p}<0,05$ & $\mathrm{p}<0,05$ \\
\hline Germen líquido pleural & $\mathrm{p}<0,05$ & NS & NS & NS \\
\hline Cultivo polimicrobiano & $\mathrm{p}<0,05$ & NS & NS & NS \\
\hline Staphylococcus aureus & $\mathrm{p}<0,05$ & $\mathrm{p}<0,05$ & $\mathrm{p}<0,05$ & NS \\
\hline Streptococcus spp & NS & NS & NS & NS \\
\hline Pseudomonsa aeruginosa & $\mathrm{p}<0,05$ & NS & $\mathrm{p}<0,05$ & $\mathrm{p}<0,05$ \\
\hline Klebsiella pneumoniae & $\mathrm{p}<0,05$ & $\mathrm{p}<0,05$ & NS & NS \\
\hline Escherichia coli & NS & NS & NS & NS \\
\hline Acinectobacter baumannii & $\mathrm{p}<0,05$ & NS & NS & $\mathrm{p}<0,05$ \\
\hline Otro germen & NS & NS & NS & NS \\
\hline Tipo cirugía & NS & NS & NS & NS \\
\hline Reoperación & $\mathrm{p}<0,05$ & $\mathrm{p}<0,05$ & NS & NS \\
\hline UCI & $\mathrm{p}<0,05$ & $\mathrm{p}<0,05$ & $\mathrm{p}<0,05$ & $\mathrm{p}<0,05$ \\
\hline
\end{tabular}


rotomía (drenaje pleural) hasta la decorticación pleuropulmonar por toracotomía con resección pulmonar. Desconocemos el número de pacientes que en el mismo período, desarrollaron un EPN en los cuales no se realizó algún procedimiento quirúrgico. Por lo anterior, si bien no representa a todos los pacientes con EPN, es una serie quirúrgica numerosa y constituye una de las mayores comunicadas en nuestro país.

El EPN es la principal causa de empiema pleural en nuestra serie total y corresponde al 70,6\% de los casos. Fue más frecuente en el género masculino y la edad promedio fue similar a otras series quirúrgicas de $\mathrm{EPN}^{11-14}$.

Las comorbilidades como diabetes mellitus, tabaquismo y cualquier condición que provoque deterioro de la inmunidad, se consideran factores que se asocian al desarrollo de EPN y a morbimortalidad de éste ${ }^{5,7,8,15,16}$. En esta serie, además de los factores mencionados destaca que el 7,4\% de los pacientes desarrollaron el EPN en el contexto de una neoplasia maligna (pleuropulmonar o de otro tipo) y que un número no menor, presentaban factores que se asocian a gravedad como deterioro de conciencia o hemodinámico (con requerimiento de drogas vasoactivas al momento de la cirugía).

En las series comunicadas, la neumonía adquirida en la comunidad (NAC) es la que más se asocia a EPN ${ }^{2,4,5}$, lo mismos sucede en la nuestra y en particular con la de tipo III de la clasificación de la American Thoracic Society. La mayoría de estas neumonías fue diagnosticada y tratada en otros hospitales antes de ser derivadas a nuestro centro con EPN.

Una de las características importantes al evaluar los empiemas es el tiempo de evolución, que permite clasificarlos según su etapa evolutiva y así determinar conducta y pronóstico ${ }^{1,2,4,15,17}$. En nuestra serie, esta variable no fue consignada por lo complejo e impreciso que resultaría obtener estos resultados en la revisión retrospectiva. El hecho que la mayoría de los pacientes fueron derivados de otros centros, con lo que suponemos que llevaban varios días o semanas con tratamiento de la neumonía antes de llegar al procedimiento quirúrgico y, que la mayoría de los pacientes necesitó una decorticación por toracotomía, nos hacen inferir que un alto porcentaje se encontraba en fase de organización o en etapa III.

Esto explicaría el bajo número de pacientes en quienes se realizó cirugía videotoracoscópica o video-asistida, que es el abordaje de elección en muchos casos de empiema, en particular en etapas iniciales. Otra posible consecuencia de esto, es que en esta serie, los cultivos microbiológicos positivos de líquido pleural son llamativamente bajos, alcanzando sólo el 23,6\%. El hecho que se trate mayoritariamente de empiemas en fase de organización y que llevan varias semanas con tratamiento antibiótico podrían explicar en parte estos resultados. En las series publicadas de EPN entre el 50 a 70\% de los cultivos de líquido pleural son positivos y los gérmenes más frecuentemente aislados son Staphylococcus aureus y Streptococcus spp ${ }^{11,12,14,18}$, similar a lo encontrado en nuestra serie.

Las alternativas quirúrgicas van desde la pleurotomía hasta la decorticación por toracotomía y la elección depende de varios factores como: etapa evolutiva del empiema, factores de riesgo del paciente o experiencia y disponibilidad del equipo quirúrgico.

La pleurotomía es una alternativa de primera línea en empiemas iniciales o en casos en que la cirugía no es posible por factores como grave comorbilidad del paciente. Es simple de realizar, se requiere implementación menor y tiene un éxito entre el 65 a 75\%, por lo que se describe una necesidad de otro procedimiento quirúrgico entre el 25 a 35\% de los casos. La mortalidad fluctúa entre 10 y 25\% y los resultados son peores cuando se utiliza como terapia única en pacientes de alto riesgo o que tienen contraindicación de

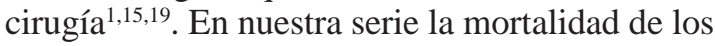
pacientes tratados exclusivamente con pleurotomía fue de 16,4\%.

La procedimientos quirúrgicos por vía videotoracoscópica o video-asistida en empiema tienen un éxito entre el 68 y 93\% de los casos, con un rango de conversión a cirugía abierta entre el 10 y 40\% especialmente en las etapas más avanzadas de empiema. La mortalidad en pacientes tratados con esta modalidad varía entre 3,5 y 5\%1,6,14,15,20,21. En nuestra serie sólo 7 (2,9\%) pacientes fueron tratados con esta técnica y las razones posibles de esto ya fueron comentadas.

La decorticación pleuropulmonar por toracotomía está indicada cuando las otras técnicas no son posibles o suficientes, y es la vía de abordaje más utilizada en empiemas en etapa avanzada. Es el procedimiento quirúrgico más invasivo y se puede asociar a resección pulmonar, resección costal u otros procedimientos. La morbilidad alcanza en algunas series hasta el 30\% y la mortalidad descrita es aproximadamente el 10\%. Las complicaciones más frecuentes son la infección de herida operatoria, fugas aéreas por fístula broncopleural y colecciones residuales que requieren re-intervención ${ }^{1,2,12,13,15,16,20,22}$.

El uso de fibrinolíticos en EPN continúa siendo controvertido, aunque tendría un rol si 
se utiliza precozmente en pacientes con EPN tabicados, particularmente en aquellos con alto riesgo quirúrgico y en centros con poca resolución quirúrgica ${ }^{1,2,4,5,15,23}$. En nuestra serie el uso de fibrinolíticos no fue una variable considerada.

La morbilidad global de los pacientes con empiema puede alcanzar entre 20 y $30 \%$ y la mortalidad descrita varía entre 3,5 y $20 \%{ }^{1-6,8,9,11-}$ $13,15,16,18,20,22$. Para nuestra serie la morbilidad fue $26,9 \%$ y la mortalidad fue $6,6 \%$.

En nuestra serie la morbilidad y la mortalidad presentaron variables asociadas en el análisis univariado y multivariado. De estas, la presencia de gérmenes considerados "agresivos" (como Staphylococcus aureus, Klebsiella pneumoniae, Acinetobacter baumannii), la edad mayor de 70 años y otras comorbildades como diabetes mellitus y “ambiente neoplásico”, también son comunicadas en otras series quirúrgicas de $\mathrm{EPN}^{15,16}$.

A modo de conclusiones podemos señalar que: el EPN es el tipo más frecuente de empiema pleural; los tratados quirúrgicamente: son más frecuentes en el género masculino, la mayoría está asociado a NAC; sólo en un bajo porcentaje se identifican gérmenes en líquido pleural; la decorticación por toracotomía es el procedimiento quirúrgico más frecuente y el tratamiento quirúrgico no está exento de morbi-mortalidad. En nuestra serie se identificaron variables asociadas a morbilidad y a mortalidad.

\section{Agradecimientos}

A nuestras secretarias Sra. Lidia León F. y Sra. Laura Cortés A., por su constante y desinteresado apoyo en la preparación de esta comunicación.

\section{Bibliografía}

1.- MOLNAR TF. Current surgical treatment of thoracic empyema in adults. Eur J Cardiothorac Surg 2007; 32: 422-30.

2.- BRIMS F J, LANSLEY S M, WATERER G W, LEE Y C. Empyema thoracis: new insights into an old disease. Eur Respir Rev 2010; 19: 220-8.

3.- SALGUERO J, CARDEMIL G, MOLINA J, LEMBACH H, FERNÁNDEZ R. Empiema Pleural: Etiología, tratamiento y complicaciones. Rev Chil Cir 2009; 61: 223-8.

4.- LIGHT R W. Parapneumonic effusions and empyema. Proc Am Thorac Soc 2006; 3: 75-80.

5.- KOEGELENBERG C F N, DIACON A H, BOLLIGER

C T. Parapneumonic pleural effusion and empyema. Respiration 2008; 75: 241-50.

6.- AHMED A E, YACOUB T E. Empyema thoracis. Clin
Med Insights Circ Respir Pulm Med 2010; 4: 1-8.

7.- CHALMERS J D, SINGANAYAGAM A, MURRAY M P, SCALLY C, FAWZI A, HILL A T. Risk factors for complicated parapneumonic effusion and empyema on presentation to hospital with community-acquired pneumonia. Thorax 2009; 64: 592-7.

8.- PICARD E, JOSEPH L, GOLDBERG S, MIMOUNI F B, DEEB M, KLEID D, et al. Predictive factors of morbidity in childhood parapneumonic effusion-associated pneumonia: a retrospective study. Pediatr Infect Dis J 2010; 29: 840-3.

9.- BENDER J M, AMPOFO K, SHENG X, PAVIA A T, CANNON-ALBRIGHT L, BYINGTON C L. Parapneumonic empyema deaths during past century, Utah. Emerg Infect Dis 2009; 15: 44-8.

10.- AMPOFO K, HERBENER A, BLASCHKE A J, HEYREND C, PORITZ M, KORGENSKI K, et al. Association of 2009 pandemic influenza A (H1N1) infection and increased hospitalization with parapneumonic empyema in children in Utah. Pediat Infect Dis J 2010; 29: 905-9.

11.- OZOL D, OKTEM S, ERDINC E. Complicated parapneumonic effusion and empyema thoracis: microbiologic and therapeutic aspects. Respir Med 2006; 100: 286-91.

12.- CHENG G, VINTCH J R. A retrospective analysis of the management of parapneumonic empyemas in a county teaching facility from 1992 to 2004 . Chest 2005; 128: 3284-90.

13.- BAR I, STAV D, FINK G, PEER A, LAZAROVITCH T, PAPIASHVILLI M. Thoracic empyema in high-risk patients: conservative management or surgery? Asian Cardiovasc Thorac Ann 2010; 18: 337-43.

14.- LUH S P, CHOU M C, WANG L S, CHEN J Y, TSAI T P. Video-assisted thoracoscopic surgery in the treatment of complicated parapneumonic effusions or empyemas: outcome of 234 patients. Chest 2005; 127: 1427-32.

15.- COLICE G L, CURTIS A, DESLAURIERS J, HEFFNER J, LIGHT R, LITTENBERG B, et al. Medical and surgical treatment of parapneumonic effusions : an evidence-based guideline. Chest 2000; 118: 1158-71.

16.- HSIEH M J, LIU Y H, CHAO Y K, LU M S, LIU H P, WU Y C, et al. Risk factors in surgical management of thoracic empyema in elderly patients. ANZ J Surg 2008; 78: 445-8.

17.- SAHN S A. Diagnosis and management of parapneumonic effusions and empyema. Clin Infect Dis 2007; 45: 1480-6.

18.- TU C Y, HSU W H, HSIA T C, CHEN H J, CHIU K L, HANG L,W, et al. The changing pathogens of complicated parapneumonic effusions or empyemas in a medical intensive care unit. Intensive Care Med 2006; 32: 570-6.

19.- THOURANI V H, BRANDY K M, MANSOUR K A, MILLER J I JR, LEE R B. Evaluation of treatment for thoracic empyema: a cost-effectiveness analysis. Ann Thorac Surg 1988; 66: 1121-7. 
20.- CHAMBERS A, ROUTLEDGE T, DUNNING J, SCARCI M. Is video-assisted thoracoscopic surgical decortication superior to open surgery in the management of adults with primary empyema? Interact Cardiovasc Thorac Surg 2010; 11: 171-7.

21.- LUH S P, HSU G J, CHENG-REN C. Complicated parapneumonic effusion and empyema: pleural decortication and video-assisted thoracic surgery. Curr Infect
Dis Rep 2008; 10: 236-40.

22.- CASALI C, STORELLI E S, DI PRIMA E, MORANDI U. Long-term functional results after surgical treatment of parapneumonic thoracic empyema. Interact Cardiovasc Thorac Surg 2009; 9: 74-8.

23.- SAHN S A. Diagnosis and Management of paraneumonic effusions and empyema. Clin Infect Dis 2007; 45: 1480-6.

Correspondencia a:

Dr. Roberto González Lagos.

Instituto Nacional del Tórax, $4^{\circ}$ piso,

Sección de Cirugía de Tórax. José Manuel Infante 717,

Providencia, Santiago, Chile.

Fono-fax: 056-02-5754997

E-mail: rgonzalezlagos@udec.cl 